\title{
Infancias y colecho en la Argentina: factores sociodemográficos, socioeconómicos y de salud familiar
}

\author{
lanina Tuñón, Ph. D. ${ }^{a}$ \\ Universidad Católica Argentina, Observatorio \\ de la Deuda Social Argentina, Argentina \\ Carolina Emilia Martínez, Lic. ${ }^{b}$ \\ Universidad Católica Argentina, Observatorio \\ de la Deuda Social Argentina, Argentina
}

ianina_tunon@uca.edu.ar

\section{Resumen (analítico)}

Se caracteriza el fenómeno del colecho en términos sociodemográficos, socioeconómicos y en relación con las vulnerabilidades específicas de los hogares, tales como la violencia intrafamiliar y los consumos nocivos. A través de la Encuesta de la Deuda Social Argentina y un análisis multivariado cuantitativo, se abordan los siguientes interrogantes: ¿el colecho es una práctica asociada únicamente a los bebés?, ¿resulta más frecuente en contextos de pobreza o se trata de una práctica que atraviesa a diferentes infancias? Se concluye que el colecho no es un fenómeno exclusivo de los y las bebés; que en la adolescencia es más regresivo para las mujeres y que el factor socioeconómico remite a un «colecho forzoso» que se especifica en interacción con el hacinamiento, la monoparentalidad, los consumos nocivos y la violencia intrafamiliar.

\section{Palabras clave}

Infancia, desarrollo del niño, relación padres-hijos, crianza del niño, bienestar de la infancia, desigualdad social, familia monoparental, demografía, salud.

\section{Thesauro}

Tesauro de Ciencias Sociales de la Unesco.

\section{Para citar este artículo}

Tuñón, I. \& Martínez, C. E. (2022). Infancias y colecho en la Argentina: factores sociodemográficos, socioeconómicos y de salud familiar. Revista Latinoamericana de Ciencias Sociales, Niñez y Juventud, 20(1), 1-21.

https://dx.doi.org/10.11600/rlcsnj.20.1.4312

\section{Historial}

Recibido: 06.04.2020

Aceptado: 22.02.2021

Publicado: 12.11.2021

\section{Información artículo}

Este artículo de investigación se desprende del proyecto denominado «La pobreza infantil en la Argentina (2010-2019): un enfoque multidimensional en perspectiva de derechos humanos», código 2017-0061, financiado por la Agencia Nacional de Promoción Científica y Tecnológica a través del Fondo para la Investigación Científica y Tecnológica, Proyectos de Investigación Científica y Tecnológica Orientados. Investigadora responsable: Dra. lanina Tuñón. El proyecto de investigación inició el 25 de abril de 2019 y finaliza el 31 de diciembre de 2021. Área: ciencias sociales, otras ciencias sociales. Subárea: ciencias sociales, interdisciplinaria 


\section{Bedsharing and childhood: socio-demographic, socio-economic and family health factors}

\section{Abstract (analytical)}

This paper analyses the phenomenon of bed-sharing in terms of sociodemographic and socioeconomic variables, but also considers specific vulnerabilities like domestic violence and addictions. It is worth examining whether this practice is associated only with babies, or else if vulnerable contexts also have an impact on children's and adolescent's co-sleeping. The information was obtained through Social Debts in Argentina Survey, and this quantitative research concludes that in this country not only early years bed-share, the phenomenon impairs teenage girls more often and that the socioeconomic factor plays a decisive role. For this reason, we propose the term "forced-bed-sharing", which refers to when co-sleeping is intensified by variables like overcrowding, single-parent family type, addictions and domestic violence.

Keywords

Childhood, social inequality, social structure, single parent families, demography, health.

\section{Co-leito na infância: fatores sócio-demográficos, socioeconômicos e de saúde familiar}

\section{Resumo (analítico)}

O artigo caracteriza o fenómeno do co-leito considerando variáveis sócio demográficas e socioeconómicas, mas também vulnerabilidades específicas como a violência doméstica ou vícios. Neste contexto, perguntamo-nos: associa-se só aos bebés? Tem mais frequência em condições de vulnerabilidade? Esta prática tem um impacto ou motivações diferentes em relação ás características das crianças? A informação foi obtida através do Questionário da Dívida Social Argentina, e o desenho de pesquisa quantitativo conclui que não é somente um fenómeno exclusivo da primeira infância, na adolescência é mais frequente em meninas e que o fator socioeconómico é decisivo. Por isso, propomos o termo "co-leito forçoso", que refere a quando a regularidade da cama compartilhada se intensifica por variáveis como a superlotação, o tipo de família monoparental, vícios e violência doméstica.

\section{Palavras-chave}

Infância, desigualdade social, estrutura social, família monoparental, demografia, saúde.

\section{Información autoras}

[a] Licenciada en Sociología. Magíster en Investigación en Ciencias Sociales. Doctora en Ciencias Sociales de la Facultad de Ciencias Sociales de la Universidad de Buenos Aires (Argentina). Coordinadora del Barómetro de la Deuda Social de la Infancia, Pontificia Universidad Católica Argentina. (iD) 0000-0002-9862-0878. Índice: H:14.

Correo electrónico: ianina_tunon@uca.edu.ar

[b] Licenciada en Psicología de la Facultad de Psicología de la Universidad de Buenos Aires (Argentina). Candidata a Doctora en Psicología de la Facultad de Psicología de la Universidad de Buenos Aires. Becaria doctoral del Conicet. (iD) 0000-0002-3006-713X.Correo electrónico: carolina_martinez@uca.edu.ar 


\section{Introducción}

$\mathbf{E}_{\text {promocionada por una vasta literatura destinada a padres y madres en proceso de }}$ crianza, y acompañada de una proliferación de ofertas de cunas colecho en el mercado. ${ }^{2}$ El fomento de esta práctica - y de otras como el amamantamiento- se apoyaría en sus ventajas para el desarrollo del apego seguro (Bowlby, 1989). De acuerdo con Gozzo-Perretti y Oliveira (2017), el colecho se presenta como una oportunidad para prolongar el contacto de los padres y las madres con su bebé, en un contexto en el que se ha extendido la inserción sociocupacional de las mujeres.

Sin desconocer este reciente auge, lo cierto es que el colecho constituye una práctica ancestral y controvertida tanto en el campo académico como en el ámbito de las publicaciones de divulgación, tal como se evidencia en las diferentes maneras de definirlo y en las diversas recomendaciones para que sea seguro. Una revisión de la literatura permite advertir que esta práctica suele relacionarse con la muerte súbita, la frecuencia y el momento del día en que tiene lugar, así como con la compañía en que se practica (padres, madres, pares u otras personas). También se registran valoraciones distintas según las edades del niño o la niña (si es o no prematuro o prematura, si ya pudo o no dormir solo o sola), así como en función de si se comparte colchón, sofá o una cuna anexada a la cama. Asimismo, en relación con los riesgos para la vida del bebé, se consideran diversos factores, tales como la presencia excesiva de almohadones o de arropamiento, una superficie para dormir blanda, la ubicación de la cama respecto de la pared, los problemas de salud de los adultos como la obesidad o las adicciones (Critzmann, 2019; Landa-Rivera et al., 2012; Landowsky et al., 2017; Vázquez, 2017).

\footnotetext{
${ }^{1}$ En este estudio se entiende por colecho a la práctica por la cual bebés, niños, niñas y adolescentes duermen con uno o dos progenitores en una misma cama o colchón, o con otros adultos de referencia del hogar.

2 A modo ilustrativo, pueden consultarse los artículos de Elbebe.com y Kellymom (2019), así como las ofertas disponibles en Amazon.
} 
Frente a los enfoques que señalan la ventaja del colecho para el desarrollo del apego seguro, un contrapunto relevante se encuentra en la teoría psicoanalítica y en la noción de la conformación del aparato psíquico. De acuerdo con este enfoque, el proceso de constitución y subjetivación del niño y de la niña involucra su diferenciación respecto de su entorno (su madre, su padre y el mundo exterior). Es así que la adquisición de autonomía requiere de la experiencia de la distancia que lo distingue (lo que se entiende por primera frustración) y del desarrollo de la capacidad de espera (distintos a la satisfacción inmediata), entre otros (Allende \& Bardi, 2017; Emmerich \& Finocchiaro, 2015). En este sentido, el hábito de dormir se aprende y representa para el niño y la niña un momento de separación (diferenciación) con respecto a sus padres (Allende \& Bardi, 2017).

En este marco, se destaca la perspectiva cultural como reproducción de prácticas y saberes populares. Al respecto, Keller y Goldberg (2004) proponen considerar la existencia o no de un vínculo de cohesión entre la actitud y las creencias de los adultos. Tal como advierten las autoras, están quienes creen que las niñas y niños deben dormir con compañía, quienes afirman que siempre deben dormir solos y aquellos que no pueden evitar esta costumbre a pesar de sostener que deben dormir solos.

Por su parte, Horsley et al. (2007) publicaron una revisión bibliográfica sistemática en la que establecieron las ventajas y desventajas del colecho. El valor de esta publicación reside en que agruparon la información con un criterio común y, al no haber un consenso sobre la definición del colecho, partieron de concebirlo como una práctica en la que adultos y niñas o niños de entre o y 2 años comparten una misma superficie para dormir. Así se abre un interrogante acerca de los factores que inciden en que las infancias de tres a 17 años compartan cama o colchón.

Asimismo, resulta relevante caracterizar a los hogares en los que es más probable la práctica del colecho; en particular, en relación con los aspectos estructurales de sus condiciones de vida. Tal como señalan Horsley et al. (2007) y Rossato (2019) se ha registrado una cantidad cada vez mayor de muertes infantiles a causa del síndrome de muerte súbita del lactante (SMSL), las cuales ocurren en mayor medida en familias con bajo nivel socioeconómico. En este mismo sentido, resulta fundamental reunir información sobre el ambiente en el que duermen los niños y niñas, así como sobre las variables que podrían especificar el fenómeno y aportar a la determinación de los principales factores de riesgo.

Chapur et al. (2019) y Horsley et al. (2007) explican que el diagnóstico de muerte súbita inesperada infantil (MSII) contiene tanto el diagnóstico del síndrome de muerte súbita infantil como las causales de ahogamiento, inhalación de contenido gástrico, 
obstrucción del tracto respiratorio por ingestión, diagnósticos equívocos, muertes sin asistencia y muertes mal definidas; en cambio, las causas médicas o forenses quedan por fuera de esta categoría. A partir estos antecedentes, entre 1991 y 2014, en la Argentina se estimó que un $7 \%$ de las defunciones infantiles se debieron a MSII y que la causa más frecuente a nivel nacional fue el SMSL. Por su parte, un estudio estadounidense (Kemp et al., 2000) que indagó las condiciones en que 119 bebés murieron durante el sueño, diagnosticó que 88 casos fueron por SMSL, 16 por sofocación y en 15 casos fue indeterminado. De acuerdo con los hallazgos del estudio, el $61.1 \%$ de los y las bebés fue encontrado en posición prona; $75.9 \%$ estaba durmiendo en una superficie no apta para la edad; $29.4 \%$ tenía cubierta la cara o cabeza con la cobija y $47.1 \%$ compartía la superficie para dormir. Solamente el $8.4 \%$ fue encontrado en una posición no prona y sin compañía, con la cabeza o cara destapada.

Los antecedentes aquí presentados manifiestan la relevancia de indagar sobre el fenómeno del colecho. Cabe considerar que, si bien la mayor parte de los estudios se focalizan en los primeros meses o años de vida, existe evidencia de que es una práctica extendida en etapas subsiguientes, durante la edad escolar y la adolescencia (Tuñón, 2019, 2020) y que supone una vulneración de derechos. En este sentido, los antecedentes de la cuestión invitan a profundizar sobre el fenómeno del colecho desde una perspectiva multidimensional que considere las características macro y microcontextuales de los hogares. Cabe pensar que estas características podrían estar asociadas al fenómeno con diferencias según la edad de la niña y el niño, sobre todo teniendo en cuenta las desigualdades sociales en la Argentina, las cuales se replican de manera similar en otros países de la región latinoamericana.

Más específicamente, el artículo se propone abordar el siguiente interrogante: ¿el colecho se trata de un fenómeno restringido a los primeros meses de vida y guarda independencia de factores sociodemográficos como el tipo de hogar y el sexo, e incluso de la situación socioeconómica de los hogares? Tal como se señala en la literatura, existen factores que predisponen al colecho, tales como los consumos nocivos de los adultos (alcohol o drogas); en efecto, se sostiene que estas conductas son en sí mismas determinantes de dicha práctica o mantienen una relación con la condición socioeconómica de los hogares. También se conjetura que la propensión a la violencia intrafamiliar puede constituirse en un factor probablemente correlacionado con aspectos estructurales de los hogares. En este marco, uno de los aportes relevantes del presente artículo es la perspectiva más amplia del fenómeno en términos del ciclo vital de las infancias, los procesos necesarios de 
constitución como sujetos distintos de los padres (que en algunos casos son transitados por las partes con ansiedades de diversa índole) y su relación con múltiples factores, tales como sociodemográficos, estructurales de las condiciones de vida de las familias y relacionados con las vulnerabilidades específicas asociadas a conductas nocivas de los adultos de referencia.

\section{Método}

El presente artículo se basa en un diseño de investigación de tipo cuantitativo a través de encuestas por muestreo. Para obtener datos representativos a nivel nacional se trabajó con los microdatos de la Encuesta de la Deuda Social Argentina (EDSA), relevada por el Observatorio de la Deuda Social Argentina del Departamento de Investigación Institucional de la Universidad Católica Argentina, cuya medición se realiza de modo periódico todos los años sobre una muestra de aglomerados urbanos de 80 ooo habitantes. ${ }^{3}$ Sobre la base de estos datos, se realizaron análisis descriptivos mediante tablas bivariadas, multivariadas y modelos de regresión logística.

La muestra utilizada para realizar el estudio corresponde al tercer trimestre del 2017 y del 2018, y está conformada por 10934 niños y niñas que tienen entre o y 17 años. Los puntos muestrales son 96o radios censales, correspondientes al Censo Nacional de Población, Hogares y Viviendas 2010. El tipo de muestreo es polietápico por conglomerados y estratificado. Se estima un error muestral total de $+/-0.93 \%$, con una estimación de una proporción poblacional del 50\% y un nivel de confianza del $95 \%$.

La EDSA es una encuesta multipropósito que incluye un módulo específico con indicadores de desarrollo humano y social de las infancias, el cual es respondido por el principal adulto de referencia del niño o niña del hogar. El instrumento contempla el registro de indicadores a nivel de cada uno de los niños y niñas del hogar entre o y 17 años con residencia habitual en el mismo. ${ }^{4}$

\footnotetext{
${ }^{3}$ El relevamiento incluye las siguientes áreas: área metropolitana del Gran Buenos Aires, Ciudad Autónoma de Buenos Aires y zo partidos del Gran Buenos Aires, Gran Rosario, Gran Córdoba, San Miguel de Tucumán y Tafí Viejo, Gran Mendoza y San Rafael, Mar del Plata, Gran Salta, Gran Paraná, Gran Resistencia y Goya, Gran San Juan, Neuquén-Plottier-Cipolletti, Zárate, La Rioja, Comodoro Rivadavia y Ushuaia-Río Grande.

${ }^{4}$ Pueden consultarse los formularios de la EDSA en el sitio web de la Universidad Católica Argentina, ingresando al Observatorio de la Deuda Social Argentina.
} 
En este estudio, la variable dependiente es la propensión por compartir cama o colchón para dormir. Operativamente se mide a través de la siguiente pregunta: «iComparte (el niño o la niña) cama o colchón para dormir?». Este indicador es medido en el marco de la EDSA de modo periódico desde el año 2010 y registra robustez en términos de su fiabilidad y convergencia con indicadores sociodemográficos y socioeconómicos (Tuñón, 2020). A continuación, se presentan las variables consideradas en el análisis como independientes: sociodemográficas, estructurales y que representan vulnerabilidades específicas de los hogares y los niños y las niñas (tablas 1 a 3 ).

Tabla 1

Indicadores sociodemográficos

\begin{tabular}{lcc}
\hline \multicolumn{1}{c}{ Variable } & \multicolumn{1}{c}{ Descripción } & Categorías \\
\hline \multirow{3}{*}{ Edad } & Se analizan las diferencias por grupos de edad de las infancias. & De 0 a 2 años \\
& & De 3 a 5 años \\
& & De 6 a 11 años \\
Sexo & Se analiza la propensión al colecho en niños y en niñas. & De 12 años \\
& & Varón \\
\multirow{2}{*}{ Tipo familiar } & $\begin{array}{l}\text { Conformación familiar, núcleo conyugal completo con hijos e hijas y } \\
\text { núcleo conyugal incompleto con hijos e hijas. }\end{array}$ & Monoparental \\
\hline
\end{tabular}

Tabla 2

Indicadores referidos a las características del contexto de tipo estructural

\begin{tabular}{llc}
\hline Variable & \multicolumn{1}{c}{ Descripción } & Categorías \\
\hline \multirow{3}{*}{ Estrato socioeconómico } & $\begin{array}{l}\text { Considera los principales activos del grupo doméstico en dos nive- } \\
\text { les: los propios del hogar (acceso a bienes y servicios) y los referi- } \\
\text { dos al jefe económico (el máximo nivel de educación alcanzado y la } \\
\text { situación ocupacional). }\end{array}$ & $\begin{array}{c}\text { Bajo } \\
\text { Hacinamiento }\end{array}$ \\
\cline { 2 - 2 } & $\begin{array}{l}\text { Niños, niñas y adolescentes en viviendas con cuatro personas o más } \\
\text { por cuarto habitable. }\end{array}$ & Sí́ \\
& & No alto \\
\hline
\end{tabular}




\section{Tabla 3}

\section{Indicadores referidos a vulnerabilidades especificas de los hogares}

\begin{tabular}{llc}
\hline \multicolumn{1}{c}{ Variable } & \multicolumn{1}{c}{ Descripción } & Categorías \\
\hline Agresión física & $\begin{array}{l}\text { Hogares en los que se utiliza la agresión física como forma } \\
\text { de disciplinar. }\end{array}$ & Sí \\
Agresión verbal & $\begin{array}{l}\text { Hogares en los que se utiliza la agresión verbal como forma } \\
\text { de disciplinar. }\end{array}$ & Sí \\
Alcoholismo en el hogar & $\begin{array}{l}\text { Establece la dependencia de algún miembro del hogar al } \\
\text { alcohol durante el último año. }\end{array}$ & Sí \\
Drogas en el hogar & $\begin{array}{l}\text { Establece la dependencia de algún miembro del hogar a las } \\
\text { drogas durante el último año. }\end{array}$ & No \\
Niños o niñas con disca- & Nodica la existencia de alguna discapacidad permanente en \\
pacidad & el infante, con o sin certificado que lo ratifique. & Sí \\
\hline
\end{tabular}

\section{Resultados}

\section{Factores sociodemográficos de las familias que practican el colecho}

Se estima que dos de cada diez niños, niñas y adolescentes en la Argentina urbana comparten cama o colchón para dormir (22.7\%). Asimismo, se advierte que aumenta la probabilidad de colecho a medida que desciende la edad. En efecto, el grupo entre o y 2 años registra el doble de probabilidad que los adolescentes de entre 12 y 17 años. No obstante, es destacable que el 15\% de este último grupo de edad duerme con alguien habitualmente (figura 1).

En la figura 1 y en la tabla 4 se puede observar que, conforme los niños y niñas son más grandes, la tendencia a dormir en camas separadas es ascendente, independientemente del sexo o del tipo de configuración familiar. Asimismo, si bien en general no hay diferencias significativas según el sexo, las adolescentes mujeres tienen más propensión al colecho que sus pares varones (tabla 4 ).

\footnotetext{
${ }^{5}$ Landa-Rivera et al. (2012) señalan que, a inicios de la primera década de este siglo, varios países reportaban cifras similares de práctica de colecho en lactantes menores de tres meses, entre ellos Irlanda (21\%), Alemania (23\%), Italia (24\%), Escocia (25\%), Austria (30\%); y otros países cifras mucho mayores, como en Suecia (65\%). Asimismo, señalan que las cifras de colecho han aumentado con el incremento de la prevalencia de la lactancia materna.
} 
Figura 1

Porcentaje de niños y niñas entre o y 17 años que comparten cama o colchón

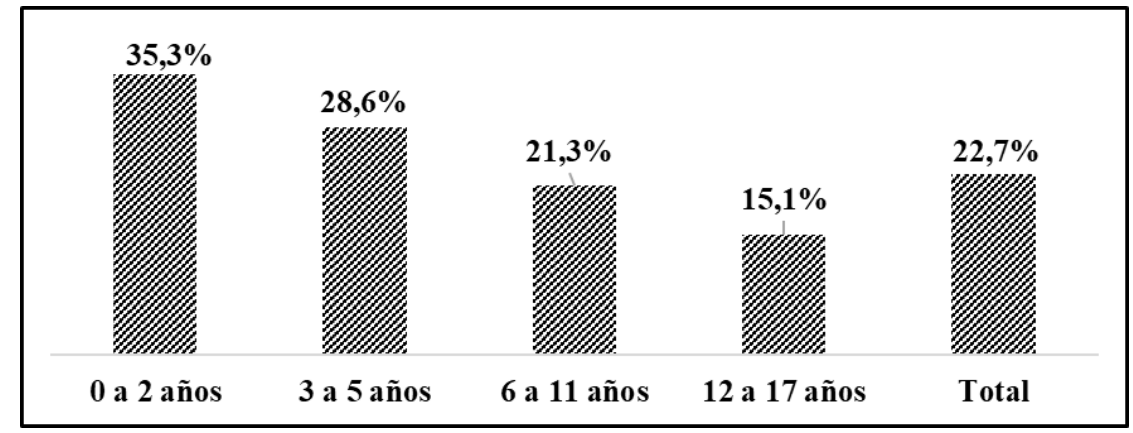

\section{Tabla 4}

Porcentaje de niños y niñas entre o y 17 años que practican colecho por grupo de edad según características sociodemográficas

\begin{tabular}{|c|c|c|c|c|c|c|}
\hline & & \multicolumn{4}{|c|}{ Grupo de edad } & \multirow{2}{*}{ Total } \\
\hline & & 0 a 2 & 3 a 5 & 6 a 11 & 12 a 17 & \\
\hline \multirow{2}{*}{ Sexo } & Varón & 36.50 & 28.80 & 20.90 & 12.10 & 21.80 \\
\hline & Mujer & 34.10 & 28.30 & 21.80 & 18.10 & 23.60 \\
\hline \multirow{2}{*}{ Tipo familiar } & Biparental & 31.60 & 25.90 & 19.60 & 14.30 & 21.00 \\
\hline & Monoparental & 49.10 & 38.80 & 27.60 & 17.30 & 28.60 \\
\hline Total & & 35.30 & 28.60 & 21.30 & 15.10 & 22.70 \\
\hline
\end{tabular}

A su vez, se advierte que sin distinción de edad hay mayor probabilidad de compartir cama o colchón para dormir en los hogares monoparentales que en los biparentales. No obstante, es importante señalar que dicha disparidad disminuye a medida que aumenta la edad (tabla 4 ).

\section{Factores estructurales de los hogares que practican el colecho}

La tabla 5 muestra que hay mayor propensión al colecho a medida que los hogares son más vulnerables en términos de sus condiciones sociales estructurales: $37 \%$ en el estrato social muy bajo ( $25 \%$ más pobre) y $38 \%$ en hogares en situación de hacinamiento. Así, se evidencia que la brecha de desigualdad social es regresiva para los grupos con menores recursos, quienes registran más del triple de probabilidad de compartir cama o colchón 
que sus pares en el estrato social más rico (25\% superior). ${ }^{6}$ No obstante, preciso advertir que uno de cada diez niños y niñas del estrato con más recursos socioeconómicos comparte cama para dormir y que ello probablemente no ocurre por falta de un colchón para cada miembro del hogar.

Además, con independencia del grupo de edad, los niños, niñas y adolescentes más pobres (25\% inferior) registran mayor probabilidad de compartir cama que sus pares más ricos (25\% superior). Puede notarse que, por grupos de edad, entre el estrato muy bajo y el medio alto hay notables brechas regresivas para los más pobres, y crecientes a medida que desciende la edad ( 32 puntos porcentuales - p. p.-, 28 p. p., 27 p. p. y 15 p. p. en los grupos de edad de o a 2, 3 a 5, 6 a 11, y 12 a 17 años, respectivamente). En cuanto a los hogares con y $\sin$ hacinamiento, se observan desigualdades de 19 p. p. en niños y niñas de hasta 5 años, 25 p. p. en la segunda infancia y 11 p.p. en adolescentes. Si bien el descenso de la práctica en ambos grupos es constante a medida que crecen, las disparidades sociales son persistentes y regresivas para quienes residen en situación de hacinamiento (tabla 5 ).

\section{Tabla 5}

Porcentaje de niños y niñas entre o y 17 años que practican colecho por grupos de edad según características estructurales

\begin{tabular}{|c|c|c|c|c|c|c|}
\hline & & \multicolumn{4}{|c|}{ Grupo de edad } & \multirow{2}{*}{ Total } \\
\hline & & 0 a 2 & 3 a 5 & 6 a 11 & 12 a 17 & \\
\hline \multirow{4}{*}{ Estrato socioeconómico } & Muy bajo & 52.00 & 40.00 & 37.40 & 23.30 & 36.60 \\
\hline & Bajo & 38.30 & 34.50 & 23.50 & 18.40 & 26.30 \\
\hline & Medio bajo & 23.40 & 23.20 & 15.80 & 12.20 & 16.80 \\
\hline & Medio alto & 19.70 & 11.70 & 9.50 & 8.40 & 10.80 \\
\hline \multirow{2}{*}{ Hacinamiento } & No & 29.70 & 23.40 & 15.60 & 13.30 & 18.10 \\
\hline & Sí & 48.60 & 41.70 & 40.30 & 23.70 & 38.10 \\
\hline
\end{tabular}

Los promedios de la tabla 5 muestran que hay más propensión al colecho en situaciones de hacinamiento cuanto menos recursos económicos tiene la familia y cuanto menor es la edad del niño o la niña. Sin embargo, cabe notar que quienes están en los grupos más desfavorecidos son aquellos que tienen una sostenida mayor propensión a abandonar dicha práctica a medida que el niño o la niña es más grande, lo que podría indicar que

\footnotetext{
${ }^{6}$ Entre 2010 y 2018 se estima que dos de cada diez niños y niñas en promedio vivieron en condición de hacinamiento. Puesto en relación con el nivel socioeconómico, el sector muy bajo - entre puntas del período- pasó de $43.8 \%$ a $44.7 \%$ de niños y niñas en esa situación habitacional (Tuñón, 2019).
} 
en realidad están disconformes con la situación de tener que compartir cama o colchón para dormir; es decir, que no lo hacen por elección. Por su parte, quienes están mejor posicionados muestran menores brechas en la oscilación, sobre todo entre la segunda infancia y la adolescencia.

\section{Vulnerabilidades específicas de las familias que practican el colecho}

En la tabla 6 se puede advertir que, cuando en los hogares se registran consumos problemáticos de una sustancia, es mayor el porcentaje de niños y niñas que comparte cama para dormir que cuando no existe dicho problema de salud. Tal como se puede observar, hay mayor propensión al colecho en las familias en las que al menos una persona sufre alcoholismo o drogadicción.

\section{Tabla 6}

Porcentaje de niños y niñas entre o y 17 años que practican colecho por grupo de edad según vulnerabilidades especificas

\begin{tabular}{|c|c|c|c|c|c|c|}
\hline & & \multicolumn{4}{|c|}{ Grupo de edad } & \multirow{2}{*}{ Total } \\
\hline & & 0 a 2 & 3 a 5 & 6 a 11 & 12 a 17 & \\
\hline \multirow{2}{*}{ Agresión física } & No & 32.30 & 25.10 & 19.30 & 13.50 & 20.10 \\
\hline & Sí & 43.40 & 35.70 & 27.00 & 21.80 & 30.40 \\
\hline \multirow{2}{*}{ Agresión verbal } & No & 35.40 & 28.50 & 21.50 & 15.00 & 22.70 \\
\hline & Sí & 32.60 & 29.30 & 19.50 & 16.80 & 22.00 \\
\hline \multirow{2}{*}{ Alcohol en el hogar } & No & 34.40 & 28.20 & 21.00 & 14.90 & 22.30 \\
\hline & Sí & 62.30 & 42.60 & 32.60 & 25.90 & 39.60 \\
\hline \multirow{2}{*}{ Drogas en el hogar } & No & 34.60 & 28.40 & 21.20 & 14.80 & 22.40 \\
\hline & Sí & 54.10 & 37.30 & 25.80 & 25.00 & 33.00 \\
\hline \multirow{2}{*}{ Discapacidad en el hogar } & No & 35.00 & 29.10 & 21.30 & 15.20 & 22.80 \\
\hline & Sí & 50.00 & 10.90 & 20.80 & 13.10 & 18.00 \\
\hline
\end{tabular}

Por otra parte, al evaluar las prácticas disciplinarias utilizadas en los hogares, también se observan disparidades en el porcentaje de niños y niñas que practican el colecho. Tal como evidencia la tabla 6 , quienes viven en hogares en los que se utiliza la agresión física como forma de disciplinar registran mayor propensión a compartir cama o colchón para dormir y las brechas son similares en los cuatro grupos de edad. No obstante, no sucede lo mismo en hogares donde se utilizan las agresiones verbales, ya que no hay una diferencia 
significativa de la proporción del colecho en presencia o ausencia de dicho método de disciplinamiento.

Asimismo, se puede observar que de o a 2 años tienen más probabilidad de colecho cuando el niño o la niña tiene una minusvalía, pero no aumenta la propensión a mayor $\operatorname{edad}^{7}($ tabla 6$)$.

\section{La propensión al colecho en un espacio multidimensional}

Los análisis presentados hasta aquí ofrecen una aproximación de tipo descriptiva de los principales factores asociados al colecho en la infancia. Dadas las limitaciones de este tipo de análisis, a continuación se presenta un análisis multivariado que permite identificar los factores ${ }^{8}$ que más inciden en la probabilidad de colecho en la infancia y la adolescencia, manteniendo constante el resto de los aspectos considerados. Los resultados de los modelos de regresión logística permiten estimar la fuerza y el sentido de una serie de atributos sobre la probabilidad de practicar colecho. Cabe advertir que en el modelo de regresión logística se han incluido un conjunto de atributos (sociodemográficos, estructurales, intrafamiliares y de vulnerabilidades especificas) que explican el 77.9\% de la propensión a la práctica del colecho en niños, niñas y adolescentes entre los o y 17 años.

En la tabla 7 se puede advertir que pertenecer al estrato socioeconómico más bajo es el factor que más influye en que se practique el colecho; en rigor, puede notarse que hay una pendiente creciente y constante a medida que disminuye el estrato socioeconómico de la familia. Es decir, el colecho ocurre en mayor medida en aquellos hogares con menores posibilidades de contar con bienes y servicios y en los que, además, la situación ocupacional y el nivel educativo jefe o jefa son los más frágiles. De hecho, un niño o niña en estas condiciones tiene tres veces más chances de compartir cama o colchón para dormir

\footnotetext{
${ }^{7}$ Una investigación realizada en Suiza explica cuál es la prevalencia y los determinantes del colecho en niños y niñas en edad escolar con discapacidad. Según la mayoría de los padres de niños y niñas con alguna minusvalía, lo que causa el colecho son los cuidados especiales que precisan de noche, sobre todo en casos de dificultades severas o cuando además tienen enfermedades como epilepsia o serios problemas visuales. La investigación evidencia que también deciden dormir juntos por los temores de que algo malo pueda suceder con relación a la condición de su hijo; si bien los ritmos de sueño de los padres no son buenos, son peores cuando duermen en habitaciones independientes (Jacquier \& Newman, 2017). Concomitantemente, una mejor calidad de sueño debido al colecho y temores también fueron registrados en casos en que no hay ningún tipo de discapacidad (Gozzo-Perretti \& Oliveira, 2017).

${ }^{8}$ Las variables excluidas por no tener significación estadística fueron la agresión verbal como método de disciplinamiento, la presencia de problemas de drogadicción en el hogar y la discapacidad del niño.
} 
que un par de la clase media alta. ${ }^{9}$ Esto se condice con el hecho de que esta práctica también se presente con mayor frecuencia en situación de hacinamiento. En efecto, las probabilidades aumentan casi dos veces más para aquellos en hogares en que los que el espacio no es suficiente para todos los convivientes.

En cuanto a las características familiares, se advierte mayor propensión al colecho a medida que el infante tiene menor edad, tal como fue señalado previamente. No obstante, si bien el grupo de los más pequeños tiene 2.5 veces más probabilidades de compartir cama para dormir que pares de mayor edad (según se indica en la figura 1), lo hace un $15.1 \%$ de estos últimos. Esto representa una proporción elevada teniendo en cuenta que se vulnera el derecho a la privacidad, la dignidad de la persona, con probables efectos en el sueño, el descanso y la salud física y psicológica. Asimismo, es relevante señalar que la propensión es mayor en las mujeres que en los varones, y ello ocurre especialmente en la adolescencia (tabla 7 ).

El tipo de configuración familiar también es un factor determinante del colecho. En efecto, los niños y las niñas en hogares monoparentales tienen 1.3 veces más probabilidades de compartir cama o colchón para dormir que sus pares de hogares biparentales (tabla 7). Por otra parte, si bien la tabla 6 indica que en la mayoría de los casos en que hay una vulnerabilidad específica aumenta el colecho en el hogar, en el modelo de regresión de la tabla 7 se observa que solo la agresión física y la presencia de problemas de alcoholismo se constituyen en factores estructurantes y significativos en presencia de los otros aspectos asociados, aunque con una fuerza algo menor dentro del modelo. Es decir, que solo dos de las vulnerabilidades específicas seleccionadas en este trabajo condicionan el colecho en presencia de otros factores. Cuando a estos riesgos se suman los factores socioeconómicos y sociodemográficos (como la edad del niño o la niña, el sexo y el tipo de configuración familiar), las desventajas se incrementan, todo lo cual impide que se conforme un ambiente propicio para el desarrollo humano saludable.

\footnotetext{
${ }^{9}$ Esta afirmación podemos realizarla a través de la lectura del $\operatorname{Exp}(\mathrm{b})$ en la tabla 7. La función del Exp(b) es la de describir el comportamiento de cada variable indicando la probabilidad de que un suceso ocurra, dado un atributo determinado y manteniendo constante el resto de las variables (siempre respecto de la categoría de referencia).
} 


\section{Tabla 7}

Factores que inciden en la probabilidad de practicar colecho en la población de niños y niñas entre o y 17 años

\begin{tabular}{|c|c|c|c|c|c|}
\hline & Variables & Categorías & B & Sig. & $\operatorname{Exp}(b)$ \\
\hline \multirow{6}{*}{$\begin{array}{l}\text { Factores estructurales } \\
\text { del contexto }\end{array}$} & \multirow{4}{*}{ Estrato socioeconómico } & Muy bajo & 1.134 & * & 3.109 \\
\hline & & Bajo & 0.773 & * & 2.165 \\
\hline & & Medio bajo & 0.397 & * & 1.488 \\
\hline & & Medio alto ${ }^{c}$ & & & \\
\hline & \multirow{2}{*}{ Hacinamiento } & Sí & 0.581 & * & 1.788 \\
\hline & & $\mathrm{No}^{\mathrm{c}}$ & & & \\
\hline \multirow{8}{*}{$\begin{array}{l}\text { Características intra- } \\
\text { familiares }\end{array}$} & \multirow{4}{*}{ Edad } & De 0 a 2 años & 1.006 & * & 2.734 \\
\hline & & De 3 a 5 años & 0.678 & * & 1.971 \\
\hline & & De 6 a 11 años & 0.364 & * & 1.439 \\
\hline & & De 12 a 17 años $^{c}$ & & & \\
\hline & \multirow{2}{*}{ Sexo } & Mujer & 0.1 & $\star \star$ & 1.106 \\
\hline & & Varónc & & & \\
\hline & \multirow{2}{*}{ Tipo familiar } & Monoparental & 0.307 & * & 1.36 \\
\hline & & Biparental ${ }^{\mathrm{C}}$ & & & \\
\hline \multirow{4}{*}{$\begin{array}{l}\text { Vulnerabilidades es- } \\
\text { pecíficas }\end{array}$} & \multirow{2}{*}{$\begin{array}{l}\text { Modo de disciplinar: agresión } \\
\text { física }\end{array}$} & Sí & 0.267 & * & 1.306 \\
\hline & & $\mathrm{No}^{\mathrm{c}}$ & & & \\
\hline & \multirow{2}{*}{ Alcoholismo en el hogar } & Sí & 0.326 & ** & 1.385 \\
\hline & & $\mathrm{No}^{\mathrm{c}}$ & & & \\
\hline
\end{tabular}

Nota. (1) Porcentaje global de aciertos que explica el modelo: 77.9\%. (2) Referencias: c indica la categoría de comparación, $*$ las diferencias de proporciones entre una categoría y la categoría de referencia son significativas ( $p$-value $<0.01), * *$ las diferencias de proporciones entre una categoría y la categoría de referencia son significativas $(\mathrm{p}$-value $<0.05)$.

\section{Discusión}

Compartir una cama o colchón para dormir es una costumbre que en los primeros años de vida puede constituirse en protectora para el niño o niña, en tanto se correlaciona con mayor tiempo de amamantamiento, autorregulación del sueño, a la vez que propicia el contacto intenso y prologando del bebé con su madre o adulto de referencia (LandaRivera et al., 2012). No obstante, otros estudios señalan que hay una relación estrecha entre la alimentación por pecho, el colecho y las interrupciones en el sueño durante la noche (De Grande, 2016), así como también existen situaciones en que compartir cama puede 
ser un riesgo para el niño o la niña y cuya asociación se confirma en el presente artículo como son la presencia de consumos nocivos en los adultos de referencia.

Efectivamente, existen grandes variaciones en el proceso de maduración física y psíquica desde el nacimiento en adelante, dependiendo, a su vez, de los recursos que tenga cada familia y del nivel de salud de la persona con quien duerme el niño. Podría pensarse que, en los primeros años de vida, cuando ocurre en familias saludables, no es un riesgo y puede ser protector para el bebé.

Tal como se observa en la figura 1 y en la tabla 4, la propensión a compartir cama o colchón para dormir disminuye a medida aumenta la edad. Si bien aquí no se explicita si duermen con alguno de sus progenitores, con pares u otras personas, en estudios previos se corroboró que, de los niños y niñas entre o a 5 años que comparten cama o colchón para dormir, más de la mitad (56.5\%) duermen con la mamá y/o el papá, mientras que el $34.1 \%$ duerme con una persona menor de 15 años y el $5.3 \%$ con otras mayores a 14 años (Tuñón \& Salvia, 2008).

$\mathrm{Al}$ reflexionar sobre el colecho en el grupo de los más pequeños, la investigación de Horsley et al. (2007) sugiere que, si bien hay un vínculo entre dormir con el bebé y la duración prolongada de amamantamiento, no se puede establecer cuál de los factores es el causal. A su vez, se niega haber encontrado información que certifique que el colecho es una práctica de promoción del vínculo filio-parental. En contrapartida, Gozzo-Perretti y Oliveira (2017) afirman que dormir juntos permite que los padres se involucren más en las decisiones sobre la crianza y tengan mayor contacto con los hijos, aspectos que se están incentivando en la actualidad, en parte debido a la mayor inserción laboral de las mujeres, quienes también encuentran un momento compartido al dormir juntos. A su vez, McKenna y Gettler (2015) crearon un neologismo en inglés entre dormir y amamantar, indicando que el contacto ayuda a promover la lactancia materna. Por su parte, Bovbjerg et al. (2018), Huang et al. (2013) y Landa-Rivera et al. (2012), afirman que cuando no hay colecho, el destete ocurre antes.

Otros tres estudios realizados sobre poblaciones con diferente nivel socioeconómico demostraron que, a comparación de quienes tienen una cama propia y duermen solos, los que duermen con otra persona se despiertan más veces por noche, pero por períodos cortos de tiempo (Horsley et al., 2007), lo que sería un factor protector contra el SMSL (Horsley et al., 2007; Landa-Rivera et al., 2012; Subcomisión de Lactancia Materna y Grupo de Trabajo de Muerte Súbita e Inesperada del Lactante, 2017; Thompson et al., 2017). 
Por su parte, Fleming et al. (2015), Rossato (2019) y la Subcomisión de Lactancia Materna y Grupo de Trabajo de Muerte Súbita e Inesperada del Lactante (2017) afirman que, cuando las madres y padres que duermen con sus hijos o hijas en un sofá o sillón fuman, toman alcohol o consumen drogas, aumenta significativamente el riesgo de SMSL, sobre todo si el bebé tiene menos de tres meses, nació prematuro o tiene bajo peso. Las adicciones mencionadas aumentan las probabilidades de muerte infantil por diferentes razones. ${ }^{10}$ En referencia con ello, hay estudios recientes que indican que en Argentina hay un $27.7 \%$ de fumadores activos y un $13.3 \%$ de fumadores pasivos que conviven con niños o niñas (Rodríguez-Espínola et al., 2019).

Además, hay evidencia de que las adicciones a drogas tienen un estrecho vínculo con la violencia, las rupturas familiares y con la mala calidad de sueño: convivir en un hogar en que al menos una persona tiene problemas con el alcohol aumenta la probabilidad de dificultades al dormir un $46 \%$, mientras que en los casos de consumo de otro tipo de estupefacientes esa probabilidad aumenta al 88\% (Rodríguez-Espínola \& Bonfiglio, 2017). Estos factores relacionados con compartir cama aportan más datos asociados al riesgo que suponen en la infancia.

El fenómeno del colecho también se asocia de modo significativo con la monoparentalidad, tal como se evidencia en el presente artículo. Una aproximación cualitativa permite reconocer que este tipo de configuración familiar tanto en situación de pobreza como de riqueza aumenta la probabilidad de ocurrencia del colecho, siendo que en nuestro país la mayoría de los hogares monoparentales pertenecen a los estratos sociales más bajos y, por lo general, son de jefatura femenina (Fourcade \& Tuñón, 2015; Tuñón, 2010). Además, un estudio realizado en Brasil también muestra la tendencia de que ocurre más colecho en hogares monoparentales en los que el niño o la niña vive con la mamá, así como también tiene relación con que se conviva con la abuela materna (Issler et al., 2010).

En el caso de Argentina se advierte con claridad que compartir cama o colchón para dormir se trata de un fenómeno fuertemente asociado a la situación socioeconómica crítica de los hogares y a la vulnerabilidad habitacional (condición de hacinamiento) (tablas 5 y 7). El análisis realizado aquí sobre el vínculo significativo entre el colecho y la pobreza por grupos de edad complementa con más detalles estudios como los de Horsley et al. (2007)

${ }^{10}$ En el caso de las madres que fuman, Napierala et al. (2016) afirman que la leche es modificada en su composición: reduce sus propiedades protectoras y su volumen, conteniendo tres veces más nicotina que la encontrada en el plasma. Esto afecta la salud del bebé, ya que disminuye su predisposición a tomar leche materna y acorta los tiempos de la lactancia. 
que señalan que cada vez hay más casos de muerte súbita infantil en los sectores de bajo nivel socioeconómico.

Si bien el colecho de los padres en los primeros meses de vida es lo más frecuente y en condiciones favorables podría ser beneficioso para al desarrollo del apego del niño o la niña, se conjetura que, a medida que los niños crecen, seguir practicando colecho puede tener consecuencias nocivas. Justamente, este artículo aporta evidencia sobre la incidencia del colecho entre niños, niñas y adolescentes, y sobre la mayor probabilidad de ocurrencia entre las adolescentes mujeres que entre sus pares varones (tabla 4 ).

El análisis multidimensional realizado en este trabajo permite reconocer la preeminencia de los factores contextuales de tipo estructurales como lo son el estrato socioeconómico del hogar y la condición de hacinamiento (tabla 7). Los datos son concluyentes y convergentes, y muestran que, si bien el porcentaje de niños y niñas de contextos de alta vulnerabilidad socioeconómica que practican el colecho es mucho mayor que el de quienes están en mejores condiciones, también son los que, en la medida de lo posible, abandonan esa costumbre en mayor grado conforme son más grandes. No obstante, un adolescente del $25 \%$ más pobre tiene el triple de probabilidad de compartir cama o colchón que en un par del $25 \%$ superior. Esta brecha de desigualdad social no es menor en un país en el que se estima en el que más del $50 \%$ de las infancias pertenecen a hogares pobres y viven en condiciones habitacionales inadecuadas (Fondo de las Naciones Unidas para los Niños [Unicef], 2020; Tuñón, 2020). En estos contextos las consecuencias son relevantes de ser consideradas porque afectan la salud, el desarrollo psicosocial y vulneran el derecho a la privacidad e intimidad. Un antiguo pero valioso estudio de la Unicef (2006), que se aproximó a la perspectiva de niños y niñas entre 9 y 11 años sin cama propia, señala que ellos y ellas prefieren tener su propio espacio para el descanso y que compartir cama les modifica el sueño, les ocasiona dolores físicos, altera la capacidad de concentración en la escuela; como contrapartida, poder dormir solos es asociado a sentimientos de libertad, felicidad y capacidad de proyectarse con optimismo hacia el futuro.

Otro estudio mucho más reciente y con un abordaje clínico, analizó cinco casos (un niño de cinco años, dos preadolescentes, un adolescente y un adulto) en que el colecho se conformó como una costumbre patológica en las familias. Para poner un límite con los padres o madres, los hijos comenzaron a tener conductas agresivas o adicciones como el alcoholismo, que genera rechazo por el olor y, además, promueve la inhibición sexual. En el caso de la preadolescente, la falta de intimidad le impidió cursar el saludable proceso de individuación. A su vez, compartir cama significa el colapso de la intimidad y erotismo 
de la pareja, del cual participa el niño o la niña sin contar con la madurez para abordarlo. Con respecto a ello, cabe mencionar que la motricidad y corporeidad ya desde temprana edad son la base del desarrollo humano y una forma de expresión de la integración y constitución del individuo que deben ser vividas placenteramente (Gamboa-Jiménez et al., 2019). Sin embargo, Garciandía-Imaz y Samper-Alum (2019) en su estudio encontraron que durante el colecho se pierden los límites e identidad de los espacios y tiempos necesarios para las partes. Por consiguiente, conlleva negar que tenga consecuencias en el presente y futuro, negar la responsabilidad por estar teniendo un hábito insalubre y negar que compartir la cama pueda tener un impacto en las personas.

Por este motivo, y para finalizar, en este artículo se propone la expresión colecho forzoso como aquel que se debe principalmente a vulnerabilidades socioeconómicas (falta de recursos materiales y económicos, crecer en contextos de bajos niveles ocupacionales y educativos). Al ser esta una situación de la cual es difícil salir, esa costumbre se perpetúa por más que ya no sean bebés. Tomando el ejemplo de Argentina y los datos con que se cuenta sobre su situación de carencias actuales, este concepto teórico sirve entonces para comprender por qué los porcentajes totales de colecho son altos aún en las infancias argentinas de 3 a 17 años. Otro aporte importante de este trabajo es que brinda evidencia de que el fenómeno del colecho guarda relación con la presencia de problemas de adicciones (alcoholismo) en el hogar y con estilos de crianza negativos en los que se utiliza la agresión física como forma de disciplinar.

\section{Agradecimientos}

Las autoras agradecen a los revisores anónimos por sus sugerencias y comentarios, y destacan el apoyo del Programa del Observatorio de la Deuda Social Argentina de la Pontificia Universidad Católica Argentina, la Agencia Nacional de Promoción Científica y Tecnológica a través del Fondo para la Investigación Científica y Tecnológica y el Consejo Nacional de Investigaciones Científicas y Técnicas. Cualquier mensaje con respecto a este artículo debe ser enviado al Observatorio de la Deuda Social Argentina, Pontificia Universidad Católica Argentina, Buenos Aires, Argentina, al correo de la autora corresponsal. 


\section{Referencias}

Allende, L. N., \& Bardi, D. C. (2017). El colecho: de los discursos de moda al psicoanálisis co-sleeping. Anuario de Investigaciones, 24, 51-62.

Bovbjerg, M. L., Hill, J. A., Uphoff, A. E., \& Rosenberg, K. D. (2018). Women who bedshare more frequently at 14 weeks postpartum subsequently report longer durations of breastfeeding. Journal of Midwifery and Women's Health, 63(4), 418-424. https://doi.org/gfb4bz

Bowlby, J. (1989). Una base segura: aplicaciones clínicas de la teoría del apego. Paidós.

Critzmann, S. (2019). Hoy no es siempre. Planeta.

Chapur, V. F., Alfaro, E. L., \& Dipierri, E. (2019). Epidemiología de las muertes súbitas infantiles inesperadas en Argentina: tendencia secular y variación espacial. Archivos Argentinos de Pediatría, 117(3), 164-17o. https://doi.org/10.5546/aap.2019.164

De Grande, P. (2016). Diseñado para bebés: objetos y prácticas en el primer año de vida. Revista Latinoamericana de Ciencias Sociales, Niñez y Juventud, 14(1), 287-300.

Emmerich, A., \& Finocchiaro, J. (2015). Colecho: padres que duermen con sus hijos: una lectura psicoanalítica para una práctica en auge. V Congreso Internacional de Investigación de la Facultad de Psicología de la Universidad Nacional de La Plata, La Plata, Argentina. http://sedici.unlp.edu.ar/handle/10915/55210

Fleming, P., Pease, A., \& Blair, P. (2015). Bed-sharing and unexpected infant deaths: What is the relationship? Paediatric Respiratory Reviews, 16(1), 62-67. https://doi.org/f6x3ds

Fondo de las Naciones Unidas para los Niños. (2006). Un niño, una cama: percepciones y valoraciones desde el mundo infantil. Time Research.

Fondo de las Naciones Unidas para los Niños. (2020). La pobreza y la desigualdad de niñas, niños y adolescentes en la Argentina. Efectos del COVID-19. Time Research.

Fourcade, H., \& Tuñón, I. (2015). Consonancias y disparidades en las formas en que los niños y las niñas son cuidados, criados y socializados en sus primeros años de vida. En I. Tunón (Ed.), Desafíos del desarrollo humano en la primera infancia (pp. 305-343). Biblos.

Gamboa-Jiménez, R. A., Bernal-Leiva, M. N., Gómez-Garay, M. P., Gutiérrez-Isla, M. J., Monreal-Cortés, C. B., \& Muñoz-Guzmán, V. V. (2019). Corporeidad, motricidad y propuestas pedagógico-prácticas en aulas de educación infantil. Revista Latinoamericana de Ciencias Sociales, Niñez y Juventud, 18(1), 1-22. https://doi.org/g4kw

Garciandía-Imaz, J. A., \& Samper-Alum, J. (2019). El colecho: un espacio de ambigüedades afectivas. Revista de Psicoterapia Relacional e Intervenciones Sociales, 39, 17-34. 
Gozzo-Perretti, C., \& Oliveira, R. (2017). Os significados socioculturais da prática do co-leito. Journal of Chemical Information and Modeling, 4(2), 110-129.

Horsley, T., Clifford, T., Barrowman, N., Bennett, S., Yazdi, F., Sampson, M., Moher, D., Dingwall, O., Schachter, H., \& Côté, A. (2007). Benefits and harms associated with the practice of bed sharing: a systematic review. Archives of Pediatrics and Adolescent Medicine, 161(3), 237-245. https://doi.org/10.1001/archpedi.161.3.237

Huang, Y., Hauck, F. R., Signore, C., Yu, A., Raju, T. N. K., Huang, T. T. K., \& Fein, S. B. (2013). Influence of bedsharing activity on breastfeeding duration among US mothers. JAMA Pediatrics, 167(11), 1038-1044. https://doi.org/10.1001/jamapediatrics.2013.2632 Issler, R. M. S., Justo-Giugliani, E. R., Cauduro-Marostica, P. J., Nieto, F., Milani, A. R., Schifino-Wolmeister, A., Breitenbach-Scherer, M., Oliveira-Pires, D., Nunes-Oliveira, M., Graeff-Chagas Pinto, D., Fernandes-Sarturi, B., Silva-Smidt, L. F., \& Villetti, M. C. (2010). Coleito no primeiro semestre de vida: prevalência e fatores associados. Cadernos de Saúde Pública, 26(5), 942-948. https://doi.org/bxsjph

Jacquier, D., \& Newman, C. J. (2017). Co-sleeping in school-aged children with a motor disability: a comparative population-based study. Developmental Medicine and Child Neurology, 59(4), 420-426. https://doi.org/10.1111/dmcn.13300

Keller, M. A., \& Goldberg, W. A. (2004). Co-sleeping: Help or hindrance for young children's independence? Infant and Child Development, 13(5), 369-388. https://doi.org/bshdqd

Kemp, J. S., Unger, B., Wilkins, D., Psara, R. M., Ledbetter, T. L., Graham, M. A., Case, M., \& Thach, B. T. (2000). Unsafe sleep practices and an analysis of bedsharing among infants dying suddenly and unexpectedly: Results of a four-year, population-based, death-scene investigation study of sudden infant death syndrome and related deaths. Pediatrics, 106(3), 1-8. https://doi.org/10.1542/peds.106.3.e41

Landa-Rivera, L., Díaz-Gómez, M., Gómez-Papi, A., Paricio-Talayero, J. M., Pallás-Alonso, C., Hernández-Aguilar, M. T., Aguayo-Maldonado, J., Arena-Ansotegui, J. M., Ares-Segura, S., Jiménez-Moya, A., Lasarte-Velillas, J. J., Martín-Calama, J., \& Romero-Escós, M. D. (2012). El colecho favorece la práctica de la lactancia materna y no aumenta el riesgo de muerte súbita del lactante. Dormir con los padres. Pediatría de Atención Primaria, 14(53), 53-6o. https://doi.org/g4kx

Landowsky, T. F., Klossowski, D. G., Costa, C. da C., \& Fujinaga, C. I. (2017). Aleitamento materno e o dormir compartilhado: visão dos profissionais da saúde. Revista Interdisciplinar Ciências e Saúde, 4(1), 74-87.

McKenna, J. J., \& Gettler, L. T. (2015). There is no such thing as infant sleep, there is no such thing as breastfeeding, there is only breastsleeping. Acta Paediatrica, 105(1), 17-21. https://doi.org/10.1111/apa.13161 
Napierala, M., Mazela, J., Merritt, T. A., \& Florek, E. (2016). Tobacco smoking and breastfeeding: Effect on the lactation process, breast milk composition and infant development. A critical review. Environmental Research, 151, 321-338. https://doi.org/f88mzd

Rodríguez-Espínola, S., Bonfiglio, J. I. (2017). Adicciones y vulnerabilidad social: el consumo problemático de alcohol, factores de riesgo, grupos vulnerables y consecuencias sociales. Observatorio de la Deuda Social Argentina; Universidad Católica Argentina. https://bit.ly/3w9 $\mathrm{T}_{4} \mathrm{VG}$

Rodríguez-Espínola, S., Cicciari, M. R., Delfino, G., Filgueira, P., González Insúa, F., Paternó-Manavella, M. A., \& Rave, E. (2019). La mirada en la persona como eje del desarrollo humano y la integración social: deudas y desigualdades en la salud, los recursos psicosociales y el ejercicio ciudadano. Observatorio de la Deuda Social Argentina; Universidad Católica Argentina. https://bit.ly/3ijXRIJ

Rossato, N. E. (2019). A new approach to safe infant sleep [Editorial]. Archivos Argentinos de Pediatría, 117(6), 354-355. https://doi.org/10.5546/aap.2019.eng.354

Subcomisión de Lactancia Materna y Grupo de Trabajo de Muerte Súbita e Inesperada del Lactante. (2017). Colecho en el hogar, lactancia materna y muerte súbita del lactante: recomendaciones para los profesionales de la salud. Archivos Argentinos de Pediatría, 115(5), 105-110. http://dx.doi.org/10.5546/aap.2017.S105

Thompson, J., Tanabe, K., Moon, R., Mitchell, E., McGarvey, C., Tappin, D., Blair, P., \& Hauck, F. (2017). Duration of breastfeeding and risk of sids: An individual participant data meta-analysis. Pediatrics, $140(5)$. https://doi.org/10.1542/peds.2017-1324

Tuñón, I. (2010). Determinantes de las oportunidades de crianza y socialización en la niñez y en la adolescencia. Revista Latinoamericana de Ciencias Sociales, Niñez y Juventud, 8(2), 903-920.

Tuñón, I. (2019). Infancia(s): progresos y retrocesos en clave de desigualdad. Observatorio de la Deuda Social Argentina; Universidad Católica Argentina. https://bit.ly/3bBcVUn Tuñón, I. (2020). Condiciones de vida de las infancias pre-panemia COVID-19: evolución de las privaciones de derechos 2010-2019. Observatorio de la Deuda Social Argentina; Universidad Católica Argentina. https://repositorio.uca.edu.ar/handle/123456789/10267

Tunón, I., \& Salvia, A. (2008). Argentina 2007: condiciones de vida de la niñez y adolescencia: una mirada desde la perspectiva de los derechos. Observatorio de la Deuda Social Argentina; Universidad Católica Argentina. https://bit.ly/3EEdycm

Vázquez, C. (2017, 29 de noviembre). El colecho y sus límites: ¿hasta cuándo el bebé debe dormir con sus padres? ElDiario.es. https://bit.ly/2YaG1a5 